\title{
Krebsassoziierte Fatigue
}

\author{
Sport und Entspannung gegen chronische Erschöpfung
}

Sport, aber auch Yoga, Tai Chi, Qigong und Entspannungsübungen können Krebspatienten helfen, die Strapazen einer Strahlentherapie besser zu überstehen. Damit können Patienten einer Fatigue vorbeugen. Unter dieser quälenden Müdigkeit und Erschöpfung leiden viele Krebskranke während und nach der Krebsbehandlung - auch nach einer Strahlentherapie. Die Deutsche Gesellschaft für Radioonkologie (DEGRO) rät den Patienten, bereits zu Beginn der Strahlentherapie körperlich und mental aktiv zu werden.

Strahlentherapien verbessern bei vielen Krebserkrankungen die Überlebenschancen. Obwohl die Bestrahlung über die Jahre immer effektiver und schonender geworden ist, bekommen zahlreiche Patienten eine krebsassoziierte Fatigue. „Die

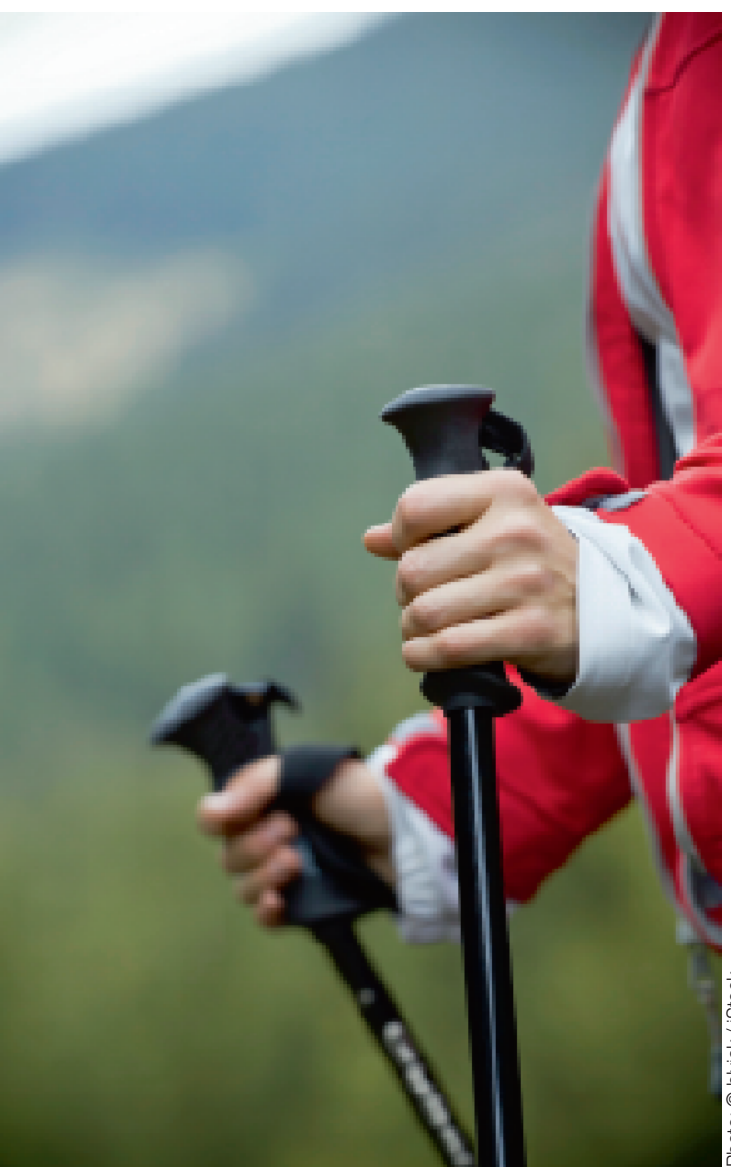

Ausdauertraining kann die durch Krebstherapie verursachte Erschöpfung mildern.
Patienten leiden unter einer großen Erschöpfung, ihre Energiereserven sind rasch verbraucht und bereits geringe körperliche Aktivitäten ermatten sie“, erklärt Prof. Dr. Stephanie E. Combs vom Klinikum rechts der Isar in München und Pressesprecherin der DEGRO. Auch Antriebslosigkeit und Konzentrationsschwierigkeiten kennzeichnen die krebsassoziierte Fatigue, unter der 60 bis 80 Prozent der Patienten nach einer Strahlen- und/ oder Chemotherapie leiden, so Combs: „Das kann so weit gehen, dass Patienten ihren Beruf nicht mehr ausüben und nicht mehr am sozialen Leben teilnehmen können."

\section{Medikamente sind wirkungslos}

Der Auslöser für Fatigue im Rahmen der Krebstherapie ist noch unbekannt. Die Behandlung ist schwierig, denn Medikamente haben sich als weitgehend wirkungslos erwiesen. „Bei manchen Patienten hilft die Einnahme von Ritalin“, so Combs: „Antidepressiva haben sich dagegen als ineffektiv erwiesen." Einige Betroffene berichteten über eine Besserung durch Ginseng oder Guaraná, andere durch verschiedene Komplementärmedizinische Verfahren, wie beispielsweise Akupunktur.

Die beste Wirkung erzielen jedoch sportliche Aktivitäten. In den vergangenen Jahren haben wenigstens 25 Studien gezeigt, dass Ausdauer- oder Kraftsport, aber auch Yoga, Tai Chi und Qigong den Krebspatienten helfen. Ebenso Walking, Radfahren und Tanzen. „Es kommt weniger darauf an, was die Patienten machen, sondern dass sie überhaupt aktiv werden", unterstreicht Combs. Einige Patienten haben jedoch geringe Motivation, da sie in der Fatigue ein Signal des Körpers sehen, sich mehr schonen zu müssen. „Sie betrachten die Erkrankung als einen Wendepunkt und finden sich mit ihrer Erschöpfungssituation ab", erklärt Combs. Deshalb helfen häufig auch psychologische Interventionen. Auch Meditation und Achtsamkeitsübungen seien empfehlenswert. Es sei wichtig, die Blockade im Kopf zu lösen und den Patienten zu zeigen, dass sich ein aktiver Lebensstil mit Bewegung für sie lohne. Viele Patienten seien jedoch be- reits offen für neue Impulse und nehmen die Empfehlungen gerne an.

\section{Anlass zum Start eines}

Bewegungsprogramms

Die Radiotherapie ist nach Ansicht von DEGRO-Präsident Prof. Dr. Jürgen Debus ein guter Anlass, mit einem Sport- oder Bewegungsprogramm zu beginnen: „Die Behandlung zieht sich häufig über mehrere Wochen. Die Patienten sind während dieser Zeit krankgeschrieben", sagt der Ärztliche Direktor der Klinik für Radioonkologie und Strahlentherapie am Universitätsklinikum Heidelberg. Die BEST-Studie des Deutschen Krebsforschungszentrums habe gezeigt, dass leichtes Krafttraining bei Brustkrebspatientinnen ab dem ersten Tag der Strahlentherapie die Fatigue und auch die Lebensqualität der Patientinnen besserte. Sie beurteilten ihre Zukunft positiver als die Teilnehmerinnen einer Vergleichsgruppe, die an einem Programm zur Muskelentspannung teilgenommen hatten.: „Weil die Radiotherapie heute so gut verträglich ist, ermutigen wir unsere Patienten zu körperlichem Training", ergänzt Debus.

Wichtig sei, dass die Patienten nicht an ihre Grenzen gingen. Sie sollten nicht mehr als 60 bis 80 Prozent ihrer Leistungsfähigkeit nutzen, denn eine zu starke Belastung könne die Fatigue verstärken.

Quelle: Presseaussendung Deutsche Gesellschaft für Radioonkologie e. V.

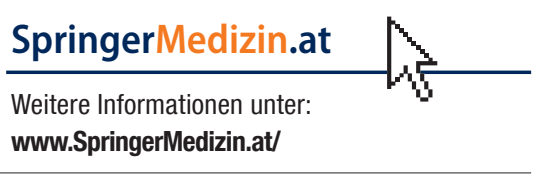

